\title{
Simulation of Bunch Precompression at High Currents in the SLC Damping Rings*
}

\author{
K.L.F. Bane, M.G. Minty, and A.W. Chao \\ Stanford Linear Accelerator Center, Stanford University, Stanford, CA 94309
}

\begin{abstract}
In the Stanford Linear Collider (SLC) each beam, after leaving a damping ring, is compressed in the Ring-to-Linac (RTL) transfer line before entering the linear accelerator. At a bunch population of $4.0 \times 10^{10}$ particles, due to the limited energy acceptance of the RTL, approximately $15 \%$ of the beam has normally been lost. During the 1996 run, however, to eliminate this loss the bunch was partially precompressed in the damping ring, just before extraction; the beam loss in the RTL was reduced to almost zero. In Ref. $(1)^{* *}$ the operation and performance of precompression are presented. Also given is an analysis which, however, does not include the effects of the longitudinal wakefield on the beam dynamics. In this report we extend that analysis to include these effects.
\end{abstract}

\section{DISTRIBUTION OF THIS DOCUMENT IS UNLIMITED}

\author{
Presented at 1997 Particle Accelerator Conference \\ Vancouver, British Columbia, Canada \\ May 12-16, 1997
}

\footnotetext{
*Work supported by Department of Energy contract DE-AC03-76SF00515.

** M. Minty, et al, "Operation and Performance of Bunch Precompression for Increased Current Transmission at the SLC," these proceedings.
}

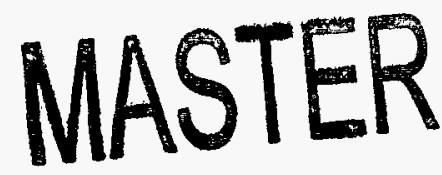




\title{
SIMULATION OF BUNCH PRECOMPRESSION AT HIGH CURRENTS IN THE SLC DAMPING RINGS *
}

\author{
K.L.F. Bane, M.G. Minty, A.W. Chao, SLAC, Stanford, CA 94309, USA
}

\begin{abstract}
In the Stanford Linear Collider (SLC) each beam, after leaving a damping ring, is compressed in the Ring-to-Linac (RTL) transfer line before entering the linear accelerator. At a bunch population of $4.0 \times 10^{10}$ particles, due to the limited energy acceptance of the RTL, approximately $15 \%$ of the beam has normally been lost. During the 1996 run, however, to eliminate this loss the bunch was partially precompressed in the damping ring, just before extraction; the beam loss in the RTL was reduced to almost zero. In Ref. [1] the operation and performance of precompression are presented. Also given is an analysis which, however, does not include the effects of the longitudinal wakefield on the beam dynamics. In this report we extend that analysis to include these effects.
\end{abstract}

\section{INTRODUCTION}

In bunch precompression in the SLC damping rings $[1,2]$ the amplitude of the rf cavity voltage $V_{r f}$ is varied over a time interval of about one synchrotron period before extraction. The beam is given two (longitudinal) kicks that generate and cancel an oscillation in the bunch centroid while, at the same time, constructively drive an oscillation in bunch length. The beam is then extracted at a minimum in bunch length. Fig. 1 displays a simulated voltage profile for bunch precompression in the SLC, one which takes into account the response time of the rf system and results in a $25 \%$ reduction in bunch length at extraction[1]. Note that $k_{0}$ in the plot is the nominal (initial) synchrotron wave number and $s / c$ is the elapsed time, with $c$ the speed of light; therefore, the abscissa is in units of the nominal synchrotron period.

In this report we begin by using multi-particle tracking to study the effects of short-range wakefields on the development of longitudinal phase space parameters, assuming the voltage profile of Fig. 1. The wakefield used in the simulations[3] has been shown to give results that are in reasonably good agreement with measurements $[4,5]$. We then apply a moment analysis to the problem-something that was also done in Ref. [1]-but now including wakefields. In this report we focus on two questions: (i) What is the validity of the moment approach in the presence of wakefields? To better explore this we will allow the beam to continue to oscillate beyond the extraction time, and compare the moment results with those obtained by tracking. (ii) How do the wakefields affect the properties of the extracted beam in the SLC?

*Work supported by the Department of Energy, contract DE-AC03$76 S F 00515$

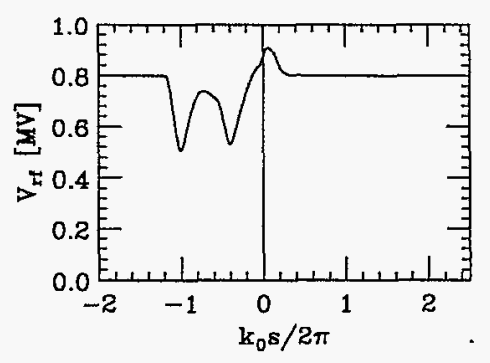

Figure 1: Cavity voltage amplitude in the SLC damping rings for bunch precompression with $N=4 \times 10^{10}$ particles per bunch[1]. The bunch is extracted at $s=0$.

\section{MULTI-PARTICLE TRACKING}

Consider an electron bunch in a storage ring. Each bunch particle $i$ has relative position and energy coordinates $\left(z_{i}, \varepsilon_{i}\right)$, with $z=0$ at the rf zero crossing $(z<0$ is to the front) and $\varepsilon=0$ for an on-energy particle. On each turn the coordinates advance by

$$
\begin{aligned}
\Delta \varepsilon_{i} & =e V_{r f}^{\prime} z_{i}-U_{0}+e V_{\text {ind }}\left(z_{i}\right) \\
\Delta z_{i} & =\frac{\alpha c T_{0}}{E_{0}}\left(\varepsilon_{i}+\Delta \varepsilon_{i}\right)
\end{aligned}
$$

with $V_{r f}^{\prime}$ the slope of the cavity voltage (a negative quantity), $U_{0}$ the per turn synchrotron radiation loss, $T_{0}$ the revolution period, $\alpha$ the momentum compaction factor, and $E_{0}$ the nominal beam energy; the beam induced voltage

$$
V_{\text {ind }}(z)=-e N \int_{-\infty}^{z} W\left(z-z^{\prime}\right) \lambda_{z}\left(z^{\prime}\right) d z^{\prime}
$$

with $N$ the bunch population, $W(z)$ the Green function wakefield, and $\lambda_{z}(z)$ the longitudinal charge distribution, normalized so that its area is 1 .

Following Eqs. 1, with $V_{r f}$ varied according to the profile of Fig. 1, we track the phase space coordinates of 85,000 macro-particles numerically. We include also, however, the effects of radiation damping and quantum excitation[6]. The wakefield used is that given in Ref. [3]. As machine parameters we take: $\alpha=0.01469$, nominal (initial) cavity voltage $V_{r f 0}=0.8 \mathrm{MV}$, rf frequency is $714 \mathrm{MHz}, c T_{0}=35.3 \mathrm{~m}, E_{0}=1.19 \mathrm{GeV}, U_{0}=79.8 \mathrm{keV}$. Note that a synchrotron period is 87 turns and a damping time is 15,000 turns.

The bunch population $N=4 \times 10^{10}$, initial rms energy spread $\sigma_{\delta 0}=7.7 \times 10^{-4}$, and initial unperturbed bunch length $\sigma_{z 00}=5.5 \mathrm{~mm}$. Solving the Haissinski Equation [7] we obtain the initial bunch shape and induced voltage (see Fig. 2). The rms length of this distribution $\sigma_{z 0}=6.7 \mathrm{~mm}$; the higher mode losses $U_{h m l}=-e^{2} N\left\langle V_{i n d}\right\rangle=76.8 \mathrm{keV}$. This bunch shape agrees well with measurements[8]. Note 
that the SLC damping ring impedance can be characterized as a resistive impedance, i.e. one for which

$$
V_{\text {ind }} \approx-c e N R \lambda_{z} \quad \text { [a resistive impedance], }
$$

with resistance $R=880 \Omega[3]$ (the dashed curve in Fig. 2). Eq. 3 is not used in the tracking simulations.

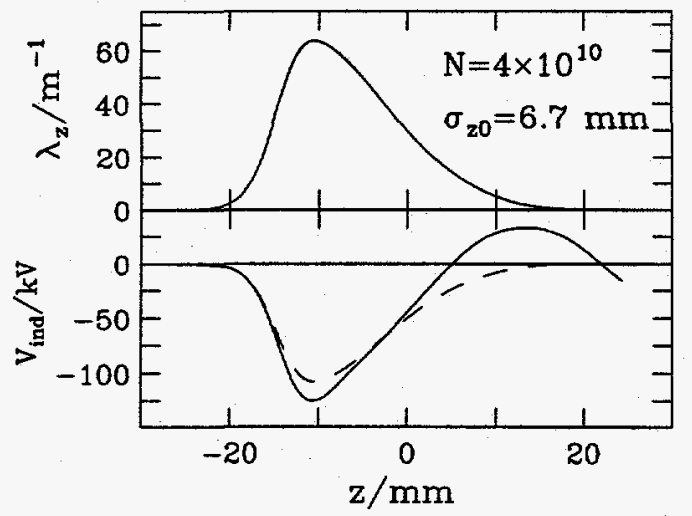

Figure 2: The initial bunch distribution used in the simulations, and the induced voltage. Also shown is Eq. 3 with $R=880 \Omega$ (the dashes).

Note that the beam current is 2.5 times the threshold to the microwave instability. We have two remarks: (i) The microwave instability in the SLC is a weak instability, with a growth time much larger than a synchrotron period[9]. Since precompression requires about a synchrotron period, the behavior of the instability is not significantly affected by bunch precompression. (ii) A time dependent feature of the observed instability, the so-called "saw-tooth" behavior, which involves the movement of a few percent of the beam[9], is not reliably seen in our simulations, and will not be addressed in this report.

\section{MOMENT EQUATIONS}

We can write second order differential equations for the moments in position and energy of the bunch distribution [ 1 , 10]. Each moment equation, however, contains as driving terms moments of $V_{i n d}(z)$, terms which themselves depend on higher moments of the distribution, and the system does not close. It will be shown, however, that since precompression occurs rather quickly, before higher order distortions become significant, and since the damping ring impedance is largely resistive, approximate solutions to the first and second moment equations can be obtained which agree quite well with tracking results.

Eqs. 1 can be written as two coupled, first-order differential equations:

$$
z^{\prime}=\alpha \delta \quad, \quad \delta^{\prime}=\left[-k^{2}(s) z-u_{0}+v(z, s)\right] / \alpha,
$$

with the relative energy deviation $\delta=\varepsilon / E_{0}$, the square of the synchrotron wave number $k^{2}=-\alpha e V_{r f}^{\prime} /\left(c T_{0} E_{0}\right)$, the normalized synchrotron radiation loss $u_{0}=\alpha U_{0} /\left(c T_{0} E_{0}\right)$, and normalized induced voltage $v=\alpha e V_{\text {ind }} /\left(c T_{0} E_{0}\right)$. Note that $k$ and $v$ depend explicitly on $s$ : the former due to the fact that the applied rf voltage changes with time, the latter because $v$ depends on $\lambda_{z}$, which also changes with time. The moment equations given below are based on Eqs. 4.

\subsection{First Moments}

The first moments are given by

$$
\langle z\rangle^{\prime \prime}+k^{2}\langle z\rangle=-u_{0}+\langle v\rangle \quad, \quad\langle\delta\rangle=\langle z\rangle^{\prime} / \alpha \quad,
$$

where the brackets signify taking the average over all particles in the bunch. Note that $\langle v\rangle=-\alpha U_{h m l} /\left(c T_{0} E_{0}\right)$; therefore, in the initial, steady state the centroid is given by $\langle z\rangle=\left(U_{0}+U_{h m l}\right) /\left(e V_{r f}^{\prime}\right)$. For the special case of a resistive wake at steady state it can be shown that

$$
\langle v\rangle \approx-\frac{\alpha e^{2} N R}{2 \sqrt{\pi} T_{0} E_{0} \sigma_{z}} \quad \text { [a resistive impedance] }
$$

with $\sigma_{z}$ the rms bunch length (discussed below).

We expect Eq. 6 to continue to be valid away from steady state, early in the precompression process. To confirm this we plot in Fig. 3a the development of $\langle v\rangle \sigma_{z}$ during precompression as obtained by tracking, and note that it deviates only gradually from its initial value. In our moment calculations we will take Eq. 6 to be valid throughout. To obtain $\langle z\rangle$ we first find $\sigma_{z}$ (as described below), then substitute Eq. 6 into Eq. 5, and solve.
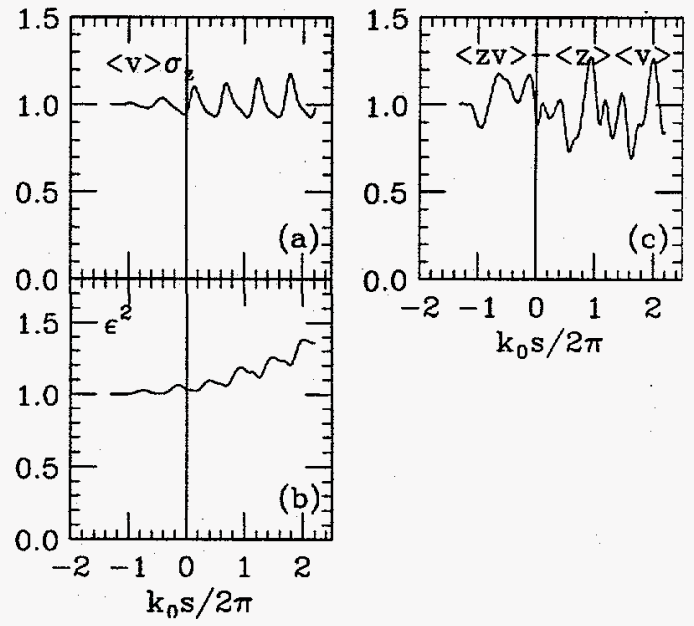

Figure 3: The evolution of three bunch parameters during precompression, as obtained by tracking [(b),(c) are discussed below]. All plots are normalized to begin with 1 .

\subsection{Second Moments}

The rms bunch length is given by

$$
\sigma_{z}^{\prime \prime}+k^{2} \sigma_{z}=\frac{\epsilon^{2}}{\sigma_{z}^{3}}+\frac{\langle z v\rangle-\langle z\rangle\langle v\rangle}{\sigma_{z}},
$$

with the emittance defined as

$$
\epsilon=\alpha \sqrt{\sigma_{z}^{2} \sigma_{\delta}^{2}-\sigma_{z \delta}^{2}}
$$


where $\sigma_{\delta}$ and $\sigma_{z \delta}$ are the second moments in $\delta$ and $z \delta$ with respect to the centroid. Note, however, that with wakefields $\epsilon$ is not a constant of motion whenever $v(z)$ depends nonlinearly on $z$, which is the case here. In Fig. 3b we plot the development of $\epsilon^{2}$ during precompression as obtained by tracking; we note that it changes only gradually, and at extraction has hardly changed at all. In the moment calculations we will take $\epsilon$ to remain fixed at its initial value.

The development of the term $\langle z v\rangle-\langle z\rangle\langle v\rangle$ in Eq. 7 during precompression, as obtained by tracking, is shown in Fig. 3c. The relative size of this term compared to $\epsilon^{2} / \sigma_{z}^{2}$ is $(40 \pm 25) \%$. For the steady state bunch distribution with a resistive impedance $\langle z v\rangle-\langle z\rangle\langle v\rangle$ depends linearly on the skew of the distribution $S_{z}$, and we might expect this to continue to be approximately true during precompression. The tracking results, however, do not give such a simple result. In solving Eq. 7 to find $\sigma_{z}$ we will simply approximate this term to be constant. For the initial conditions to be steady-state requires that constant to be

$$
\langle z v\rangle-\langle z\rangle\langle v\rangle=\alpha^{2} \sigma_{\delta 0}^{2}\left(\sigma_{z 0}^{2} / \sigma_{z 00}^{2}-1\right) .
$$

\section{RESULTS}

We begin by plotting in Fig. 4 the development of $\langle z\rangle$ and $\sigma_{z}$ during precompression assuming no wakefields, obtained by tracking (the solid curves) and by the moment approach (the dashes). Note that the plot of $\sigma_{z}$ is normalized to the nominal (no potential well distortion) bunch length $\sigma_{z 00}$. Note also that at extraction $(s=0)$ the slopes of both $\langle z\rangle$ and $\sigma_{z}$ are zero by design.
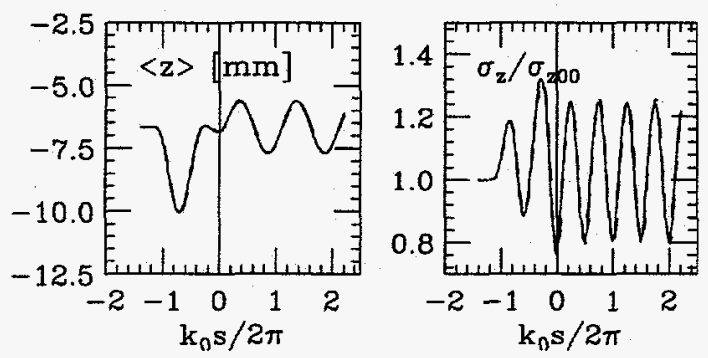

Figure 4: The development of $\langle z\rangle$ and $\sigma_{z}$ during precompression assuming no wakefields, as obtained by tracking (solid curves) and by the moment calculations (the dashes).

In Fig. 5 we plot the results when wakefields are included. In the upper part the first 2 moments in $z$ and $\delta$, as obtained by the two methods, are given. Note that the plot of $\sigma_{z}$ is normalized to the initial bunch length $\sigma_{z} 0$. The results of the two methods agree quite well. In the bottom frames of Fig. 5 we plot the skew moments as obtained by tracking. Note, for example, that due to the wakefields the skew in $z, S_{z}$, a parameter which is important in linac dynamics, does not change sign during phase space rotation.

Comparing to the earlier, no-wakefield results (Fig. 4) we find that with wakefields $\langle z\rangle$ begins more offset from 0 (due to the higher mode losses), has larger excursions, and, beyond $s=0$, has more irregular motion. As for $\sigma_{z}$ the relative oscillation amplitude is similar to before but the

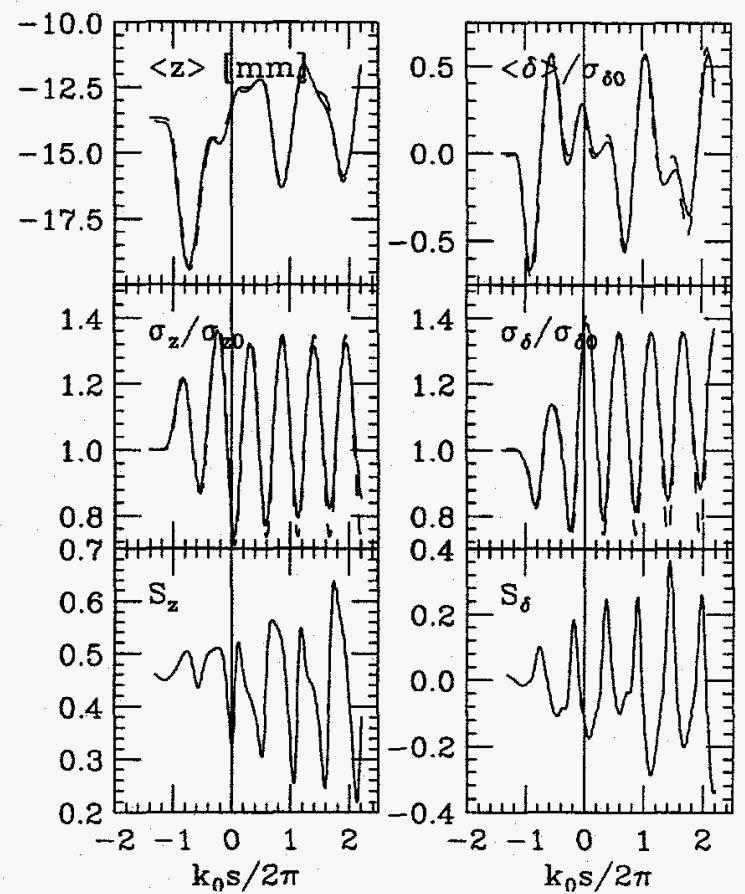

Figure 5: The development of the first 3 moments of the distribution during precompression when wakefields are included, as obtained by tracking(solid curves) and by the moment equations (the dashes). Extraction occurs at $s=0$.

phase advance lags-at $k_{0} s=4 \pi$ by $65^{\circ}$. At extraction we find that with wakefields the minimum position of $\sigma_{z}$ has moved by $k_{0} \Delta s=0.3$ and the slope of $\langle z\rangle$ is $3 \mu \mathrm{r}$. This effect, however, is not important for the SLC since the rf timing can be kept steady to $0.5 \mathrm{~ns}$, and a $0.5 \mathrm{~ns}$ drift results in a centroid shift of only $0.5 \mu \mathrm{m}$.

Finally, the implications of bunch precompression on machine stability are studied in detail in Ref. [1]. There it is pointed out, for example, that a tolerance arises from current fluctuations leading to centroid motion. Here we merely point out that at $N=4 \times 10^{10}$ a $10 \%$ change in current leads to a $0.6 \mathrm{~mm}$ change in $\langle z\rangle$ at extraction, whereas with precompression the effect is nearly twice as large.

\section{REFERENCES}

[1] M. Minty, et al, "Operation and Performance of Bunch Precompression at the SLC," these proceedings.

[2] F.-J. Decker, et al, HEEAC, Hamburg, Germany, 1992, p. 148.

[3] K. Bane, C.-K. Ng, 1993 PAC, Washington, D.C., p. 3432.

[4] K. Bane, et al, 1995 PAC, Dallas, TX., p. 3109.

[5] K. Bane and K. Oide, 1995 PAC, Dallas, TX., p. 3105.

[6] See e.g. R. Siemann, Nucl. Instr. Meth., 203, 57 (1982).

[7] J. Haissinski, Il Nuovo Cimento, 18B, No.1, 72 (1973).

[8] R. Holtzapple, SLAC-REPORT-487, PhD thesis, June 1996.

[9] B. Podobodev, private communication.

[10] A. Chao, Physics of Collective Beam Instabilities in High Energy Accelerators, (J. Wiley \& Sons, New York, 1993). 


\section{DISCLAIMER}

This report was prepared as an account of work sponsored by an agency of the United States Government. Neither the United States Government nor any agency thereof, nor any of their employees, makes any warranty, express or implied, or assumes any legal liability or responsibility for the accuracy, completeness, or usefulness of any information, apparatus, product, or process disclosed, or represents that its use would not infringe privately owned rights. Reference herein to any specific commercial product, process, or service by trade name, trademark, manufacturer, or otherwise does not necessarily constitute or imply its endorsement, recommendation, or favoring by the United States Government or any agency thereof. The views and opinions of authors expressed herein do not necessarily state or reflect those of the United States Government or any agency thereof. 


\section{DISCLAIMER}

Portions of this document may be illegible in electronic image products. Images are produced from the best available original document. 